\title{
Crystal alignment of surface stabilized polymorph in thioindigo films
}

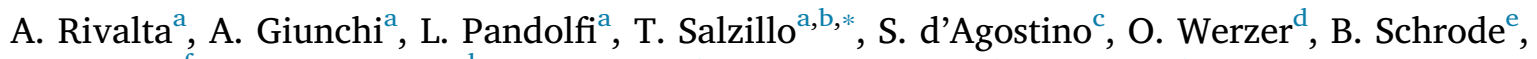 \\ N. Demitri ${ }^{\mathrm{f}}$, M. Mas-Torrent ${ }^{\mathrm{b}}$, A. Brillante ${ }^{\mathrm{a}}$, R.G. Della Valle ${ }^{\mathrm{a}}$, E. Venuti ${ }^{\mathrm{a}, * *}$ \\ a Dipartimento di Chimica Industriale "Toso Montanari”, University of Bologna, Viale del Risorgimento 4, 40136, Bologna, Italy \\ ${ }^{\mathrm{b}}$ Department of Molecular Nanoscience and Organic Materials, Institut de Ciència de Materials de Barcelona (ICMAB-CSIC) and (CIBER-BBN), Campus de la UAB, \\ 08193, Bellaterra, Spain \\ "Dipartimento di Chimica “G. Ciamician”, University of Bologna, Via F. Selmi 2, 40126, Bologna, Italy \\ ${ }^{\mathrm{d}}$ Institute of Pharmaceutical Sciences, Department of Pharmaceutical Technology, University of Graz, Universitätsplatz 1, 8010, Graz, Austria \\ ${ }^{\mathrm{e}}$ Institute of Solid State Physics, Graz University of Technology, Petersgasse 16, 8010, Graz, Austria \\ ${ }_{\mathrm{f}}^{\mathrm{f}}$ Elettra - Sincrotrone Trieste, S.S. $14 \mathrm{Km} 163.5$ in Area Science Park, 34149, Basovizza, Trieste, Italy
}

\section{A B S T R A C T}

Thioindigo (2-(3-Oxo-1-benzothiophen-2(3H)-ylidene)-1-benzothiophen-3(2H)-one) is a synthetic dye related to the natural compound Indigo. Notwithstanding the interest aroused recently by its employment as a functional material in a number of applications, a satisfactory characterization of its solid state is still missing. In this work, we study the occurrence of the two thioindigo $\alpha$ and $\beta$ polymorphs under various growth conditions, and find that their structural similarity implies they often coexist. However, whereas polymorph $\beta$ is certainly predominant in the bulk phase, polymorph $\alpha$ grows preferentially on substrates, turning out to be the surface stabilized phase in highly homogeneous and ordered films obtained by the bar-assisted meniscus shearing method (BAMS). DFT calculations support the experimental findings, aiding in the polymorph spectroscopic identification and to the interpretation of the order in the films of polymorph $\alpha$.

\section{Introduction}

Natural and synthetic indigoids comprise a large class of vat dyes, with the former used to color textiles for millennia and the latter having had industrial applications at least from the beginning of the 19th century. Like Indigo, they are all characterized by very low solubility, which makes difficult the handling of these systems in the solution of most solvents. To overcome such a problem the molecule can be transformed into a soluble species (e.g. the reduced leuco form of the Indigoids) or functionalized with groups which facilitate its dissolution. By processing the product, the starting material can be recovered either chemically or thermally. For instance, indigo and derivatives of indigowich bear ter-butilossicarbonil group (tBOC) have been used to efficiently solve the problem of forming films of these compounds, which display promising characteristics for charge transport [1]; a chemical precursor has been used to produce thioindigo in a specific polymorphic modification [2]. Further examples of similar procedures will have to be devised to extend the applications of these dyes as functional materials, bearing in mind that the fabrication process can determine or influence the phase in which the final product is obtained. In fact, if this is crystalline, its chemical-physical properties are closely related to its structural properties and therefore a sound knowledge of its polymorphism, its degree of order and the way of controlling these factors is a necessary requirement.

As a part of an extended study of the relationship between crystal structures and physical properties of organic dyes [3,4], aimed to explore their applications as functional materials in the field of organic electronics, we analyze in this work the structural properties of the synthetic indigo derivative thioindigo (2-(3-oxo-1-benzothiophen$2(3 H)$-ylidene)-1-benzothiophen-3(2H)-one), which is known to display polymorphism $[5,6]$. This compound was reported to exhibit hole field effect mobility [7], albeit smaller than indigo. Besides, the electrical properties of the silicon/thioindigo heterojunction were studied in view of applications in organic photodiodes and organic photovoltaic devices [8]. In all cases, thin films of the material were fabricated by evaporation techniques, but no information about the polymorph obtained, i.e. the solid phase which determines the final electronic properties, was given [9].

After re-determining, by X-ray crystallography, the structure of the thioindigo polymorphs, here named $\alpha$ and $\beta$, we have interpreted and rationalized their polarized Raman spectra in the low frequency range with the aid of DFT (Density functional theory) calculations. Over this range, polymorphs can be quickly and efficiently discriminated by identifying their vibrational fingerprint [10-14], a tool of the utmost importance when dealing with films. By growing micro- and nanocrystals on surfaces, using solution methods such as drop casting and

\footnotetext{
* Corresponding author. Dipartimento di Chimica Industriale “Toso Montanari”, University of Bologna, Viale del Risorgimento 4, 40136, Bologna, Italy.

** Corresponding author.

E-mail addresses: tommaso.salzillo@unibo.it (T. Salzillo), elisabetta.venuti@unibo.it (E. Venuti).
} 
the solution bar assisted meniscus shearing (BAMS) technique [15-17], we have investigated the polymorph selectivity of the substrate. We show that polymorph $\alpha$ can be obtained in oriented thin films by the BAMS technique, which has been demonstrated to produce crystalline films of excellent performance and reproducibility in the fabrication of organic field effect transistors.

\section{Experimental and computational methods}

\subsection{Crystal growth}

Thioindigo (TCI Chemicals) was purified by sublimation in low pressure environment at $250{ }^{\circ} \mathrm{C}$, obtaining microcrystals of irregular shape. Both the commercial and the sublimed crystalline powders are made of polymorph $\beta$ as checked by X-ray diffraction. Small, needle shaped crystals (about $1 \mathrm{~mm}$ long) of the same polymorph were grown from solution by slow solvent evaporation, either dichloro- and trichloro-methane. Single crystals of $\beta$ were also grown by Physical Vapor Transport (PVT). In this case, the material was placed at one end of an evacuated glass ampule, which was sealed after three cycles of evacuation and nitrogen purging. The ampule was then placed horizontally in a furnace. Two hot end temperatures were selected in distinct experiments: 250 and $285^{\circ} \mathrm{C}$, and temperature gradients of approx. $13{ }^{\circ} \mathrm{C}$ / $\mathrm{cm}$ and $15^{\circ} \mathrm{C} / \mathrm{cm}$, respectively, were measured at equilibrium. Finally, a fast sublimation near the decomposition temperature of $290{ }^{\circ} \mathrm{C}$ yielded microcrystals of regular shape of both $\alpha$ and $\beta$ polymorph in nearly equal amounts.

Crystalline non homogeneous films were obtained by drop casting saturated solutions of various solvents which were filtered $(0.2 \mu \mathrm{m}$ filter) prior to usage. All the solvents (Sigma Aldrich) used for the experiments were of spectroscopic grade. As substrates, $\mathrm{Si} / \mathrm{SiO}_{\mathrm{x}}$ wafers cleaned by an ultrasonic bath in acetone, isopropyl alcohol, and deionized water, and finally dried under nitrogen were used, providing isotropic surface properties. With most solvents (among which acetonitrile, nitrobenzene and dichloromethane) both $\alpha$ and $\beta$ polymorph crystals of a few microns were obtained, which were virtually indistinguishable in morphology but could be identified by microRaman spectroscopy in the energy range of the lattice phonon modes. Deposition from xylene yielded only the $\alpha$ form: in this case, $25 \mu$ of filtered $10^{-3} \mathrm{M}$ solution were deposited on a glass substrate kept at $60^{\circ} \mathrm{C}$, removing the excess solution by a fine tip of paper while spreading. A final set of thioindigo film samples were fabricated by BAMS on highly n-doped Si wafers with $200 \mathrm{~nm}$ of thermally grown $\mathrm{SiO}_{2}$ (from SiMat), cleaned in ultrasonic bath with acetone and isopropanol and dried with nitrogen. In the shearing method a Teflon bar was positioned above the substrate. In this case we used a Teflon bar instead of the conventional stainless steel bar because with thioindigo solution was not possible to reach the meniscus formation with the latter. The substrate was heated at $150{ }^{\circ} \mathrm{C}$ and $40 \mu \mathrm{l}$ of solution $0.5 \mathrm{mg}$ / $\mathrm{ml}\left(\right.$ ca $1.5 \times 10^{-3} \mathrm{M}$ ) were deposited at the interface and sheared at the speed of $1 \mathrm{~mm} / \mathrm{s}$. The quality of the films was found to be strongly solvent-dependent: a thioindigo saturated solution in 50/50 Benzonitrile/dimethyl formammide (v/v) yielded non homogeneous films of the pure $\alpha$ form, whereas highly textured films of the same polymorph where obtained from pure anisole. The influence of the ink formulation and coating conditions during the deposition of organic semiconductors by BAMS has already been demonstrated to have an impact on the formed polymorph $[18,19]$.

\subsection{Raman spectroscopy}

Raman spectra in the lattice phonon region $\left(10-150 \mathrm{~cm}^{-1}\right)$ were collected with a Horiba Jobin Yvon T64000 spectrometer equipped with three monochromators in double subtractive configuration. The spectrometer was coupled to an Olympus BX40 confocal microscope equipped with $100 \mathrm{x}, 50 \mathrm{x}, 20 \mathrm{x}$ and $10 \mathrm{x}$ objectives, with a lateral resolution $\approx 1 \mu \mathrm{m}$ with the $100 \mathrm{x}$ objective. Spectra were recorded with the excitation wavelength of the $647.1 \mathrm{~nm}$ line of a $\mathrm{Kr}^{+}$gas laser, reducing the power with neutral density filters to avoid sample damage due to absorption processes at this wavelength.

A half-wave plate was used to rotate the polarization of the incident light, while a wire grid polarizer selected the polarization of the scattering. X-ray indexing on crystal faces, morphology and extinction directions allowed us to orient the crystal specimens for measurements in polarized light.

\subsection{X-ray diffraction}

For $\alpha$-thioindigo, single crystal data collections were performed at the X-ray diffraction beamline (XRD1) of the Elettra Synchrotron, Trieste (Italy) [20]. Individual crystals were obtained by removing them from the substrate surface of a polycrystalline film prepared by solution deposition from acetonitrile.

The crystals were dipped in NHV oil (Jena Bioscience, Jena, Germany) and mounted on the goniometer head with a nylon loop. Complete datasets were collected at the constant temperatures of $100 \mathrm{~K}$ and $300 \mathrm{~K}$ (nitrogen stream supplied through an Oxford Cryostream 700 - Oxford Cryosystems Ltd, Oxford, UK) through the rotating crystal method on two distinct samples. Data were acquired using a monochromatic wavelength of $0.700 \AA$, on a Pilatus $2 \mathrm{M}$ hybrid-pixel area detector (DECTRIS Ltd, Baden-Daettwil, Switzerland). The diffraction data were indexed and integrated using XDS [21]. Crystals appeared as thin rods and were prone to radiation damage. The structures were solved by the dual space algorithm implemented in the SHELXT code [22]. Fourier analysis and refinement were performed by the full-matrix least-squares methods based on $\mathrm{F}^{2}$ implemented in SHELXL (Version 2018/3) [23]. The Coot program was used for modeling [24]. Anisotropic thermal motion refinements have been used for all atoms. Hydrogen atoms were included at calculated positions with isotropic $\mathrm{U}_{\text {factors }}=1 \cdot 2 \cdot \mathrm{U}_{\mathrm{eq}}\left(\mathrm{U}_{\mathrm{eq}}\right.$ being the equivalent isotropic thermal factor of the bonded non hydrogen atom). Crystals show non-merohedral twinning (reciprocal lattice two-fold twinning axis (001)), with a twin fraction of $\sim 45 \%$, identified using PLATON TWINROTMAT algorithm [25]. Twinning is responsible for distortions on heteroatoms connectivities therefore carbonyl group geometric parameters have been restrained (using SHELXL DFIX and DANG cards). Essential crystal and refinement data are reported in Table S1.

Single-crystal data for thioindigo grown by slow evaporation of a dichloromethane solution (see above for sample preparation) were collected on an Oxford X'Calibur S CCD diffractometer operated at RT and equipped with a graphite monochromator (Mo- $\mathrm{K}_{\alpha}$ radiation, $\lambda=0.71073 \AA$ ), and identified as thioindigo form $\beta$ (CSD refcode SINDIG02 [6]). Its structure was redetermined to get data with more accuracy. Data collection and refinement details are listed in Table S2. All non-hydrogen atoms were refined anisotropically. $\mathrm{H}_{\mathrm{CH}}$ atoms were added in calculated positions. SHELX97 [26] was used for structure solution and refinement on $\mathrm{F}^{2}$. The program Mercury [27] was used to calculate intermolecular interactions and for molecular graphics. Crystal data can be obtained free of charge via www.ccdc.cam.ac.uk/ structures (or from the Cambridge Crystallographic Data Centre, 12 Union Road, Cambridge CB21EZ, UK; fax: $(+44) 1223-336-033$; or email: deposit@ccdc.cam.ac.uk). CCDC numbers 1919968, 1919969, 1920128, 1920129.

\subsection{Grazing incidence $X$-Ray diffraction}

To confirm the crystallographic phase in the thioindigo films and to determine the direction of the unit cell axes with respect to the shearing direction in the BAMS deposition, grazing incidence X-ray diffraction (GIXD) measurements were performed. The beamline XRD1 (described above) was set to a wavelength of $1.4 \AA$. Diffracted intensities were recorded by the same Pilatus $2 \mathrm{M}$ detector approximately $200 \mathrm{~mm}$ after 
Table 1

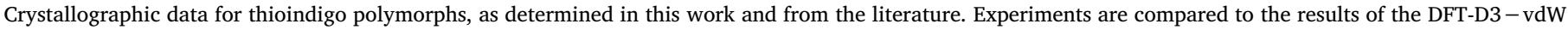
calculations also by means of the RMSD ${ }_{\geq 15}$ of the interatomic distances.

\begin{tabular}{|c|c|c|c|c|c|c|c|c|}
\hline & Space Group & $a(\AA)$ & $b(\AA)$ & $c(\AA)$ & $\beta(\operatorname{deg})$ & Volume $\left(\AA^{3}\right)$ & $\mathrm{T}(\mathrm{K})$ & $\mathrm{RMSD}_{\geq 15}(\AA)$ \\
\hline SINDIG [5] & $P 2_{1} / c$ & 7.91(3) & $3.97(2)$ & $20.41(3)$ & 93 & 640.051 & RT & \\
\hline \multirow[t]{2}{*}{ This work $\alpha$} & & $7.8130(16)$ & $3.8820(8)$ & $20.503(4)$ & $93.10(3)$ & 620.947 & 100 & 0.050 \\
\hline & & $7.8770(16)$ & $3.9390(8)$ & $20.542(4)$ & $92.67(3)$ & 636.675 & RT & 0.050 \\
\hline SINDG02 [6] & $\mathrm{P} 2{ }_{1} / \mathrm{n}$ & $3.981(3)$ & $20.65(2)$ & $7.930(7)$ & $98.84(5)$ & 644.163 & RT & \\
\hline \multirow[t]{2}{*}{ This work $\beta$} & & $3.8644(3)$ & $20.3897(14)$ & $7.8016(5)$ & $98.610(6)$ & 607.791 & 100 & 0.043 \\
\hline & & $3.9342(3)$ & $20.4464(11)$ & $7.8580(6)$ & $98.074(7)$ & 625.834 & RT & 0.045 \\
\hline This work, calc $\alpha$ & $P 2_{1} / c$ & 7.8408 & 3.8347 & 20.1538 & 93.147 & 605.053 & 0 & \\
\hline This work, calc $\beta$ & $\mathrm{P} 2_{1} / \mathrm{n}$ & 3.8481 & 20.1325 & 7.8525 & 97.539 & 603.089 & 0 & \\
\hline
\end{tabular}

the sample. To reduce the size of the incident X-ray beam on the sample (footprint), a relatively high incident angle of $1^{\circ}$ was chosen. Due to the in-plane alignment introduced by the preparation technique, the sample had to be rotated around its surface normal during the GIXD measurement to ensure that all diffraction information was collected. A total of 150 separate diffraction images were taken. Each integration was performed over $2^{\circ}$ of azimuthal sample rotation i.e. a $300^{\circ}$ azimuthal sample rotation was performed, which is sufficient to gain all the relevant information for the samples here studied. In the first step, these data allow for the determination of the polymorphs after summation of all the images. In the second step, i.e. evaluation of the inplane alignment of the crystallites, the data from the 150 images of distinct azimuths can be used to calculate pole figures for different interplanar distances. Conversion of the measured data to reciprocal space, crystal phase analysis and pole figure generation were performed using GIDVis [28]. The orientational analysis of the pole figure evaluation was performed using the software Stereopole [29].

\subsection{Computations}

DFT simulations on the two thioindigo polymorphs were performed using the code VASP (Vienna Ab initio Simulation Package) [30-33]. The Perdew-Burke-Ernzerhof (PBE) exchange correlation functional [34] was employed together with projected-augmented wave (PAW) pseudopotentials $[35,36]$. The pseudopotentials used in the calculations were PAW_PBE H (15Jun2001) and PAW_PBE C (08Apr2002). The effects of the dispersive interactions were included with the D3-BJ pairwise correction by Grimme et al. [37].

Energy convergence was achieved with identical settings for the two polymorphs: a plane wave cutoff of $800 \mathrm{eV}$ proved to be adequate in combination with $4 \times 2 \times 1$ Monkhorst-Pack k-point sampling, for the short, middle and long axis, selected to obtain the same density of points in each direction (i.e. $2 \times 4 \times 1$ and $4 \times 1 \times 2$ for $\alpha$ and $\beta$ thioindigo, respectively). Raising the cutoff energy from 800 to $1200 \mathrm{eV}$ caused energy variations around $1 \mathrm{meV} / \mathrm{atom}$, while the denser k-point sampling of $10 \times 5 \times 2$ gave energy changes below $0.05 \mathrm{meV} / \mathrm{atom}$. Lower k-point samplings actually yield the same accuracy. Atomic coordinates (or atomic coordinates and cell parameters) were fully relaxed, halting when residual forces fell below $1 \mathrm{meV} / \AA$, using the GADGET package [38]. For both energy cutoffs and k-point sampling, energy differences between polymorphs $\alpha$ and $\beta$ converge faster than absolute energies to within $0.04 \mathrm{meV} /$ atom.

Vibrational modes, restricted to the $\Gamma$ point, were computed at the experimentally determined unit cell volumes through the force constants obtained with the PHONOPY software [39] in combination with VASP. Polarizability tensors $\boldsymbol{\alpha}$ for each crystal mode were obtained by using the Python program vasp_raman.py [40], which uses the VASP code as backend. Raman intensities were finally adjusted by considering excitation wavelength and temperature dependence [41].

Depending on the orientation of the crystal with respect to the polarization of the incident and scattered light beams, the Raman intensities [42] for each given crystal vibrational mode $q$ are determined by combinations of the components of the symmetric $3 \times 3$ tensor $\alpha$, which expresses the vibrational modulation of the polarizability, i.e. the polarizability derivatives with respect to normal modes of vibration. To correlate the computed $\boldsymbol{\alpha}$ components of the tensor to the intensities measured in the Raman experiment, we need to transform the polarizability derivatives from the crystal reference frame $(a, b, c)$ to the laboratory frame. This is done by calculating the new tensor $R \boldsymbol{\alpha} \boldsymbol{R} \boldsymbol{\dagger}$, where $\boldsymbol{R}$ is the rotation matrix from one coordinate system to the other.

The orientation of a single crystal in the Raman experiment is generally specified by the Porto' notation [43], which results simplified in the backscattering experimental setup of this work, as the exciting and scattered beams propagate always in the $\mathrm{Z}$ direction of the laboratory frame. If the crystal is oriented so that at least one of its axes $a, b, c(e . g$. $a)$ is collinear with one of the laboratory axes $\mathrm{X}, \mathrm{Y}$, the only possible configurations in back scattering geometry are represented in the Porto's notation by $\mathrm{Z}(a a) \mathrm{Z}$ and $\mathrm{Z}\left(a a_{\perp}\right) \mathrm{Z}$, where $a_{\perp}$ is the direction normal to $a$. The first letter identifies the propagation direction of the excitation, while the last one refers to the observation direction. They will be dropped as redundant in the notation here adopted. The letters in parentheses specify the polarization directions of the exciting and scattered light, respectively. Besides, and more importantly, they identify the particular element of the Raman tensor responsible for the observed scattering. The mathematical expressions of the intensities resulting from the crystal orientations adopted for the thioindigo crystals can be found in the SI.

\section{Results}

\subsection{The thioindigo $\alpha$ and $\beta$ polymorphs: $X$-ray diffraction characterization} and computed structures

The structures determined for the $\alpha$ and $\beta$ thioindigo polymorphs in this work are reported in Table 1 , where the cell parameters are compared to the literature data. The table also contains the structural parameters predicted by the DFT-D3 - vdW calculations. The experimental methods used for the crystal growth of the specimen alpha and beta polymorphs are reported in detail in section 2.1.

The crystal of polymorph $\alpha$ is monoclinic $P 2_{1} / c$, with two molecules per unit cell $(Z=2)$ residing on crystal inversion centres (see Fig. 1). The molecular geometry is $\mathrm{C}_{\mathrm{i}}$, with very little deviation from a planar $\mathrm{C}_{2 \mathrm{~h}}$. Molecules are stacked along the very short $b$ monoclinic axis. Accordingly, in the projection along $b$, molecules appear lying in parallel rows on the plane $a c$ (see Fig. 2).

Polymorph $\beta$ single-crystal structure was solved and refined in the monoclinic $\mathrm{P} 2{ }_{1} / \mathrm{n}$ space group. Also in this case the monoclinic unit cell contains two molecules on inversion centres. The molecular geometry is virtually identical in the two polymorphs, but the packing of $\beta$ is quite different from that of form $\alpha$, with the molecules stacked along the shortest axis $a$ and appearing in a projection along this axis as shown in Fig. 2. In both polymorphs $\pi-\pi$ stacking interactions are exerted between the molecules aligned along the short axes of the structure, but while in the $\alpha$ polymorph all the stacks are arranged parallel to each 
<smiles>O=C1/C(=C2\Sc3ccccc3C2=O)Sc2ccccc21</smiles>

Fig. 1. Molecular structure of Thioindigo (2-(3-Oxo-1-benzothiophen-2(3H)ylidene)-1-benzothiophen-3(2H)-one).

other, in the $\beta$ neighbouring stacks are rotated with respect to each other. Note that intra-stack distances and relative molecular orientations within the stacks are identical for both structures.

At $100 \mathrm{~K}$ and room temperature, the experimentally determined volumes of the two polymorphs differ by about $2 \%$, with form $\beta$ being the denser, but the trend indicates that the density difference decreases on increasing temperature.

The information on how the previously analyzed single crystals of polymorph $\alpha$ were obtained is not reported in the literature, where this form was the first described [5]. At present, commercially available thioindigo is found to be entirely composed of polymorph $\beta$, and most of the methods used to grow bulk crystals in this work have yielded this modification. In fact, and as discussed in the following sections, polymorph $\alpha$ has been obtained only by deposition from solution, often together with polymorph $\beta$, and as a pure phase only from xylene at $60{ }^{\circ} \mathrm{C}$ and at the high deposition temperature of BAMS.

The lattice parameters computed at the minimum energy structure $(0 \mathrm{~K})$ by relaxing both the atomic coordinates and cell axes with the DFT-D3-vdW method employed in this work are reported in Table 1. Notwithstanding experimental volumes necessarily larger than the calculated ones (which do not include the thermal expansion), the agreement is very good and has been quantitatively checked by computing the RMSD $\geq 15$ of the interatomic distances [44].

In agreement with the experimental findings at finite temperature, polymorph $\beta$ is calculated to be slightly denser than polymorph $\alpha$ at $0 \mathrm{~K}$. The energy difference between the two phases $(\approx 0.02 \mathrm{kcal} / \mathrm{mol})$, however, is smaller than the computational accuracy reported for these calculations [45] and, in fact, it is impossible to reliably predict which modification is the more stable.

\subsection{Raman characterization of single crystals}

MicroRaman spectroscopy in the lattice phonon region represents a very versatile technique currently employed to diagnose the presence of different structures grown in a variety of environments, such as films, nano-crystals or at solid interfaces [46-48]. The modes detected in the low energy region of the vibrational spectrum, i.e., the lattice phonons, owe their properties to the weak dispersion forces of the intermolecular field and are therefore distinctive of a given structure.

The vibrational spectrum mirrors the lattice dynamics, rather than its structure, so that spectroscopic and structural information can be linked through the precise characterization of crystals whose structure is known by X-ray.

By lattice phonon spectroscopy we discriminate between $\alpha$ and $\beta$ thioindigo polymorphs, and establish the reference spectra characteristic of each phase. The results obtained, complemented with the Raman measurements in polarized light, contain information about and can be rationalized in terms of the structural organization of the solid phase.

In the left hand side of Fig. 3, we show the experimental Raman spectra of $\alpha$-thioindigo crystal below $200 \mathrm{~cm}^{-1}$, together with the DFTD3-vdW spectrum calculated at the room temperature experimental structure. As specified above, the spectra were measured on single microcrystals grown from thioindigo solutions in acetonitrile and xylene drop cast on substrates, having the morphologies displayed in the image of the Figure. In the right hand side of Fig. 3, we report the $\beta$ thioindigo spectra of the commercial powder, as well as those of the samples grown by the PVT method and from dichloromethane solutions. Again, the experimental profiles are compared to the DFTD3 - vdW spectrum at the experimental structure. Two markedly distinct profiles are found for the two polymorphs, making their recognition easy when analysing still unknown powders and crystals of thioindigo.
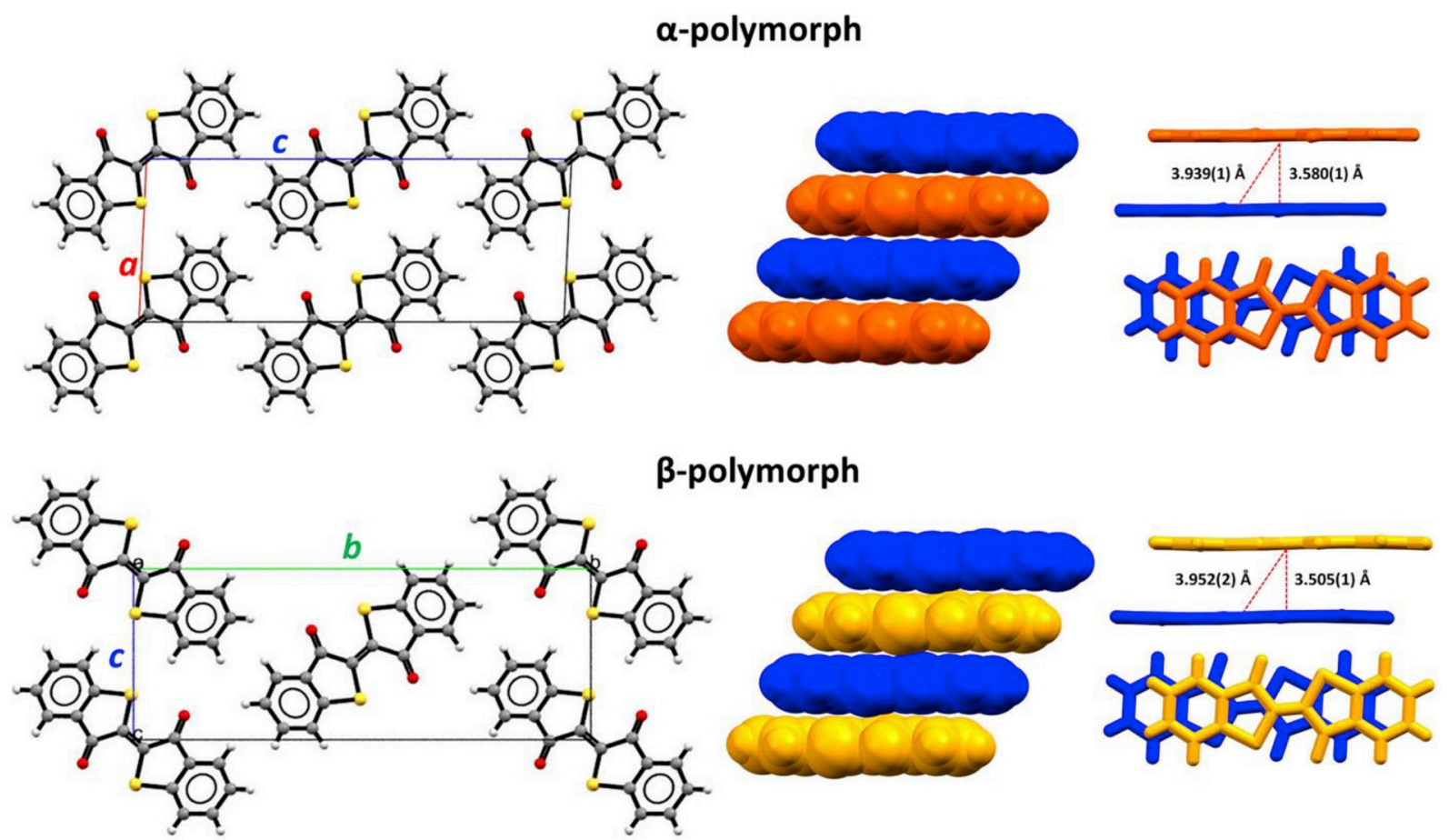

Fig. 2. Packing of the $\alpha$ - and $\beta$-indigo polymorphs; left: projection along the crystallographic axes $b$ and $a$ for polymorphs $\alpha$ and $\beta$, respectively; right: representation of the packing along the stacking direction. 

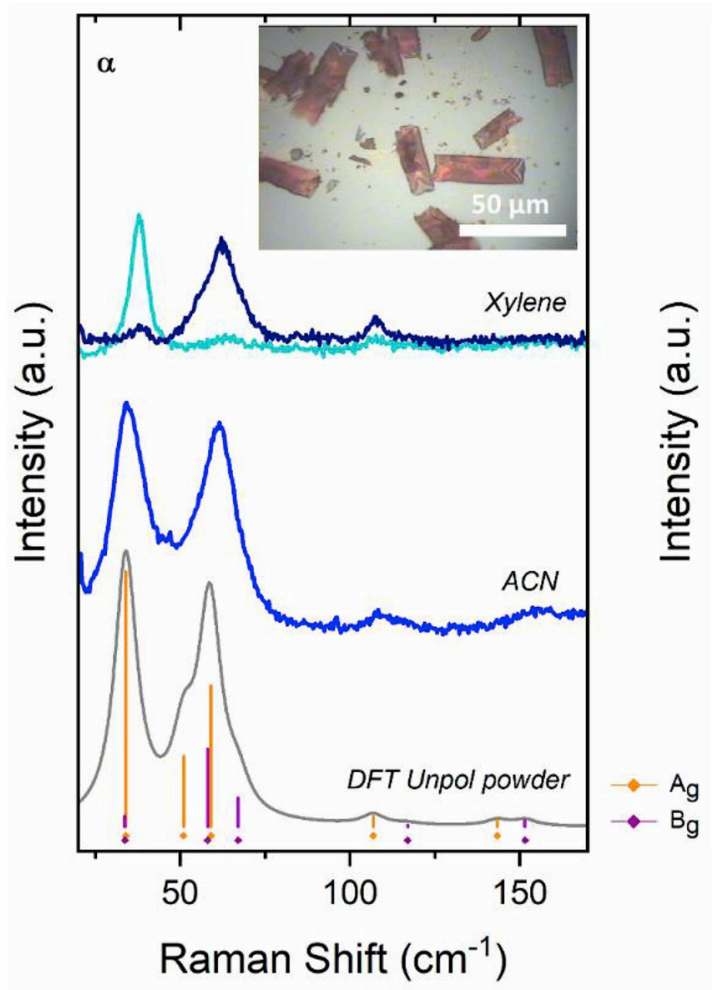

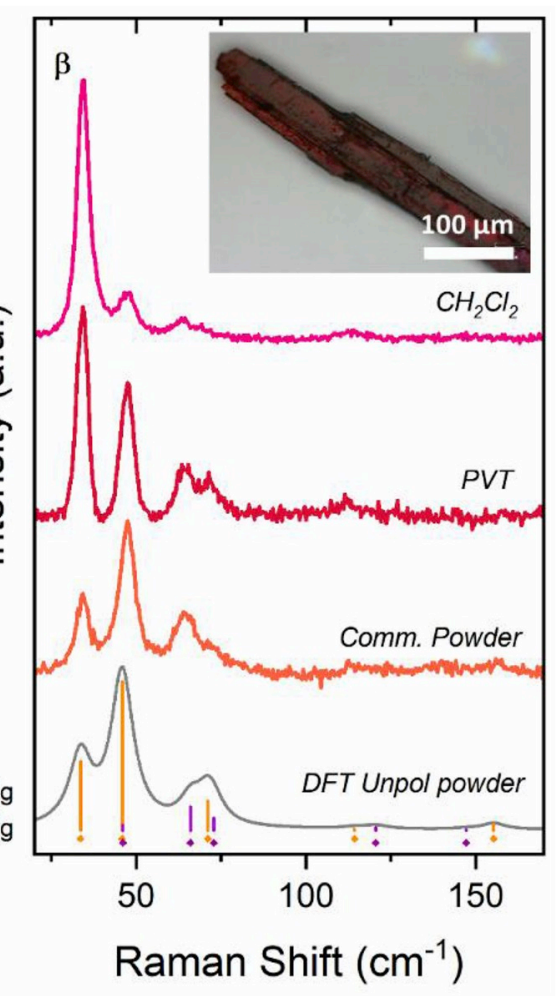

Fig. 3. Experimental and unpolarized (powder) simulated DFT-D3-vdW Raman spectra and of $\alpha$ (left) and $\beta$-thioindigo (right) crystal samples (see images in the inlets; $\alpha$ crystal obtained from xylene and $\beta$ from $\mathrm{CH}_{2} \mathrm{Cl}_{2}$ ) in the range $10-200 \mathrm{~cm}^{-1}$, where lattice phonons are observed. The spectra of $\alpha$-thioindigo from xylene in are given for two different orientations of the crystal as an instance of crystal anisotropy. Vertical bars of height proportional to the intensity of the mode identify the calculated values, with colour codes yellow and violet for modes of symmetry $A_{g}$ and $B_{g}$, as indicated. The spectral profiles are built by convolution of Lorenzian bands (see text for details). (For interpretation of the references to colour in this figure legend, the reader is referred to the Web version of this article.)
For $\alpha$-thioindigo, only six modes were calculated below $80 \mathrm{~cm}^{-1}$, whereas at the temperature of the experiment, we detect over this range two broad features. The calculations reveal that these are the superimposition of closely located peaks, which are rendered in the computed trace by the convolution of Lorentzian bands with a HWHM (half width at half maximum) of $4 \mathrm{~cm}^{-1}$, to conform to the experimental widths. The $\beta$ polymorph shows a slightly better resolved profile that, again, can be properly accounted for by the appropriate convolution of the first six calculated modes.

Table 2 reports the calculated Raman shifts in the same energy interval, along with the symmetry assignment in the framework of the monoclinic $P 2_{1} / c$ space group and the component analysis of the calculated eigenvectors. Since both structures belong to centrosymmetric monoclinic crystals with $\mathrm{Z}=2$, we expect six Raman-active lattice phonons, which correspond to the lowest wavenumber peaks. Their symmetry is $3 \mathrm{~A}_{\mathrm{g}}+3 \mathrm{~B}_{\mathrm{g}}$, and they have the character of pure librations of the unit cell molecules taken as rigid bodies, with virtually no contribution from intra-molecular vibrations. Thus, they unambiguously identify with the vibrational fingerprints of each polymorph. A number

Table 2

Wavenumbers of the six lowest energy vibrational modes of $\alpha$ and $\beta$ thioindigo polymorphs calculated the at the RT structure, along with their symmetry assignments. The lattice phonon character of each mode is given by the sum of the squared rotational components around the molecular inertia axes. As the sums all yield $\approx 100 \%$ (unity), the first six modes closely resemble rigid bodies librations.

\begin{tabular}{llllll}
\hline \multicolumn{2}{c}{ Thioindigo Polymorph $\alpha$} & \multicolumn{3}{c}{ Thioindigo Polymorph $\beta$} \\
\hline Sym & Calc $\left(\mathrm{cm}^{-1}\right)$ & $\begin{array}{l}\text { Lattice Phonon } \\
(\%)\end{array}$ & Sym & Calc $\left(\mathrm{cm}^{-1}\right)$ & $\begin{array}{l}\text { Lattice Phonon } \\
(\%)\end{array}$ \\
\hline $\mathrm{A}_{\mathrm{g}}$ & 34.1 & 100 & & & 98 \\
$\mathrm{~B}_{\mathrm{g}}$ & 33.7 & 100 & $\mathrm{~A}_{\mathrm{g}}$ & 33.6 & 100 \\
$\mathrm{~A}_{\mathrm{g}}$ & 51.0 & 98 & $\mathrm{~A}_{\mathrm{g}}$ & 45.9 & 100 \\
$\mathrm{~B}_{\mathrm{g}}$ & 58.0 & 98 & $\mathrm{~B}_{\mathrm{g}}$ & 46.1 & 96 \\
$\mathrm{~A}_{\mathrm{g}}$ & 59.0 & 98 & $\mathrm{~B}_{\mathrm{g}}$ & 66.0 & 98 \\
$\mathrm{~B}_{\mathrm{g}}$ & 67.0 & 88 & $\mathrm{~A}_{\mathrm{g}}$ & 71.1 & 98 \\
\hline
\end{tabular}

of much weaker bands are observed at higher wavenumbers in the range shown in Fig. 3. They are correctly reproduced in both energy and intensity by the DFT simulations, and correspond to intra-molecular modes, obviously common to both polymorph, as evidenced by comparison of the patterns shown in Fig. 3.

The anisotropic behavior of the $\alpha$-thioindigo single crystal is evidenced in the top left of Fig. 3, where the two traces have been recorded for two distinct orientations of the same crystal from xylene. We can better probe such an anisotropy by measuring the spectra in polarized light, an experiment that provides the information on the phonon response for each crystallographic axis, which we can thus transfer to the interpretation of the film spectra. This implies to select the polarization of both the incoming laser field and the scattered radiation, so that a specific correspondence of each phonon band to its symmetry can be determined [49].

The results of such an analysis, obtained for oriented single crystals of both phases, are shown in Fig. 4. We start our discussion with the left hand side of Fig. 4, representative of a specimen of the $\alpha$-polymorph. By $\mathrm{X}$-ray measurements, the scattering plane of the crystals of the image of Figure is found to be the crystallographic face $a b$ or (001), with $b$ being the axis of fastest crystal growth, and $a$ perpendicular to it. This information enables us to interpret the spectra measured with polarized light in backscattering geometry. The labels $(a a),(b b)$, and $(a b)$ of the Figure are used to indicate the polarization vectors of incident (first letter) and scattered (second letter) light in relation to the crystallographic axis of a sample oriented along its extinction directions [50]. The unpolarized spectrum is given as a reference in the bottom part of the Figure and describes a situation where the light beam impinges normal to the randomly oriented face (001) and no polarization discrimination is applied to the scattered light. The experiments are compared to the DFT-D3-vdW polarized spectra calculated for the given face (001).

Due to the symmetry properties of the polarization matrix, the mode analysis indicates that the spectra $(a b)$ and $(b a)$ recorded in cross polarization must be identical and display only modes of $\mathrm{B}_{\mathrm{g}}$ symmetry. On the contrary, spectra labelled $(a a)$ or $(b b)$ must give access to $\mathrm{A}_{\mathrm{g}}$ modes 


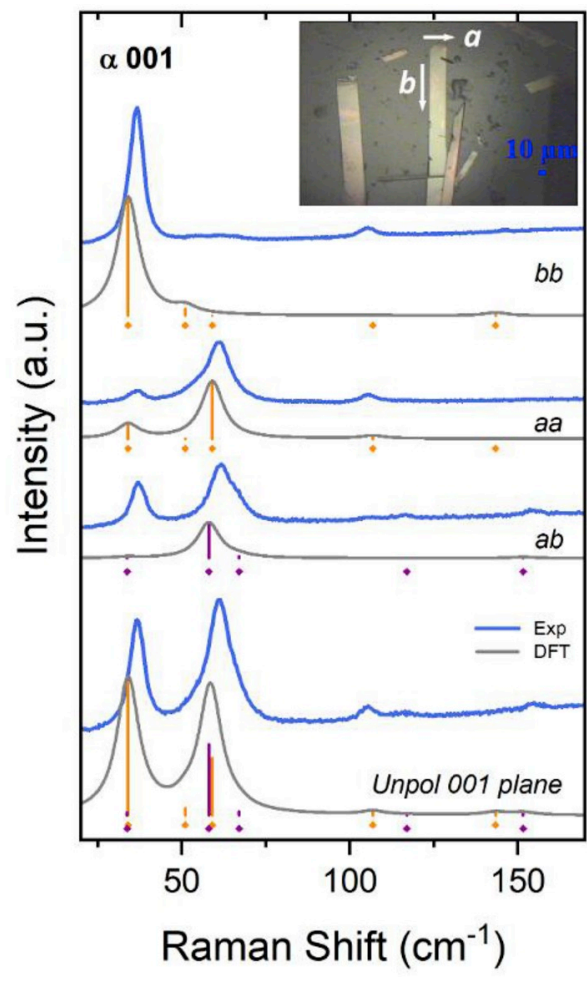

only, but the intensities in the two spectral profiles depend on the probed matrix elements, $(a a)$ or $(b b)$, respectively. These considerations allow us, for instance, to solve into an $A_{g} / B_{g}$ doublet the broad feature centred at $36 \mathrm{~cm}^{-1}$. Also, we can interpret the shift of the maximum of the other broad feature, centred around $61 \mathrm{~cm}^{-1}$, as depending on the presence of two unsolvable $\mathrm{A}_{\mathrm{g}}$ modes in the spectra labelled as $(a a)$ and $(b b)$ and two $\mathrm{B}_{\mathrm{g}}$ modes in the $(a b)$ spectra. Such an analysis is strongly assisted by the computed values and can be safely performed given the striking agreement of our theoretical model with the experiment. Notably, in this treatment, more than in the symmetry assignment of each detected mode, the interest lies into linking a specific spectral feature, and its changing intensity in polarized light, to the anisotropy properties of the crystal and to its orientation, a crucial information for the study of the films given below.

In Fig. 4, on the right, a similar analysis for the polarized Raman spectra of $\beta$-thioindigo is given for the single crystal pictured in the inset image. In the needle-like crystals of this form, the excitation beam of the Raman experiment impinges on the face (011), as probed by Xray diffraction. The needle axis identifies with the crystallographic axis $a$, and when the polarization vectors of incident and scattered light are both parallel to it (aa), only Raman-active modes of $A_{g}$ symmetry are allowed. In cross polarization, the scattering perpendicular to $a$ does not correspond to any principal component of the polarizability tensor and a mixture of $\mathrm{A}_{\mathrm{g}}$ and $\mathrm{B}_{\mathrm{g}}$ modes is expected.

\subsection{Thioindigo film characterization}

As mentioned above, thioindigo solutions deposited by drop casting mostly resulted in scarcely homogeneous specimens, characterized by the presence of nano- and micro-crystals and showing the concomitant presence of both polymorphic modifications. Notably, regardless of the solvent employed in the drop casting, the $\beta$ polymorph could never be obtained as the only phase present in the films, whereas by carefully choosing deposition conditions and solvent, like in the case of xylene, the growth of only the polymorph $\alpha$ could be induced. In fact, we could single out some high quality crystals of this form for the structural characterization by carefully removing them from the substrate. These experimental evidences hint to the selectivity of the interface for the growth of polymorph $\alpha$, and to the possibility that physically pure films of thioindigo can be obtained only with this crystal modification.

This suggested applying the BAMS growth method for the film fabrication, as this technique has been found effective in controlling film morphology and polymorphism by allowing a finer control of the crystallization kinetics and thermodynamics [15-19]. Therefore, we have tested different temperature and solvent conditions, seeking to achieve both a selective phase growth and an improved quality of the film in terms of homogeneity. X-ray diffraction was used to analyze phase and alignment in a number of selected samples, while the agile microRaman analysis, also in polarized light, was extensively used to systematically check deposition conditions by micrometric mapping, identifying those yielding a pure phase.

Fig. 5 (top left) gives the scheme of the BAMS method which was applied for the preparation of high quality films. As the liquid is dragged along the surface, the solvent evaporates at the meniscus, eventually resulting in a homogenous deposition. The optical images in polarized light under crossed polarizers suggest the presence of a macroscopic alignment of the domains along the shearing direction. Finally, the XRD pattern of the film reveals the presence of the polymorph $\alpha$ only. The contact plane corresponds to the crystal face $a b$, with the thioindigo molecules standing at the interface, and the long molecular axis forming an angle of $22^{\circ}$ with the normal to the substrate, as shown in the Figure.

To gain access to the other crystallographic directions of the BAMS film, rotating grazing incidence X-ray diffraction measurements were used. Such a method allows to construct pole figures from which the orientation of the crystal planes with respect to the shearing direction can be deduced (see SI for further details). The results show that the film clearly displays a preferential in-plane alignment. In particular, the $a$ axis is found to be parallel to the shearing direction. Optical microscopy reveals that adjacent crystals are slightly inclined in their growth direction, which is often observed when using shearing methods on isotropic surface. Using the rotating GIXD this variation is determined to be about $20^{\circ}$. Improvement to the overall growth direction might be obtained by using structured surfaces as demonstrated for similar 


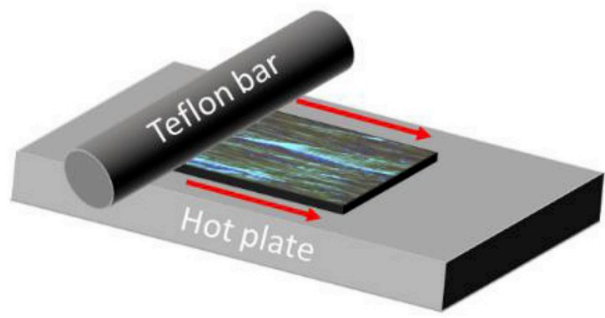

(002)

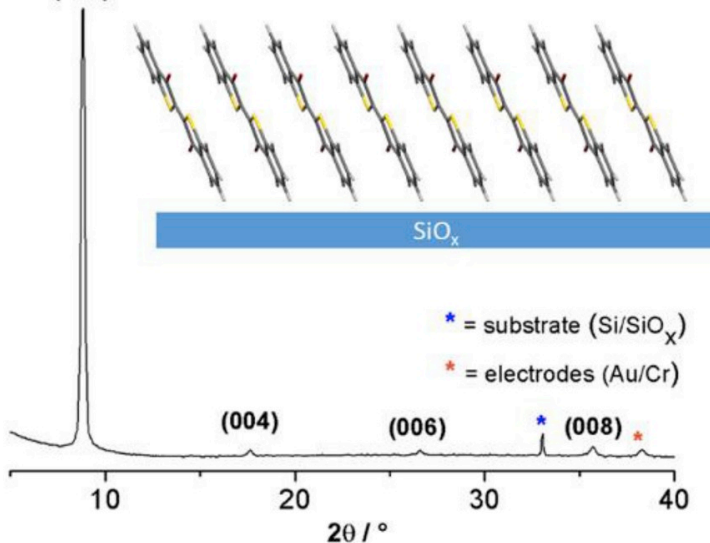

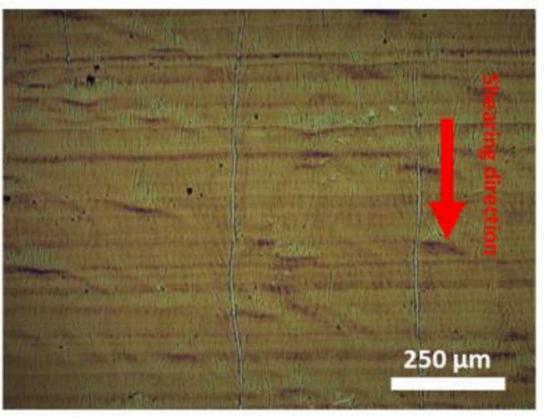

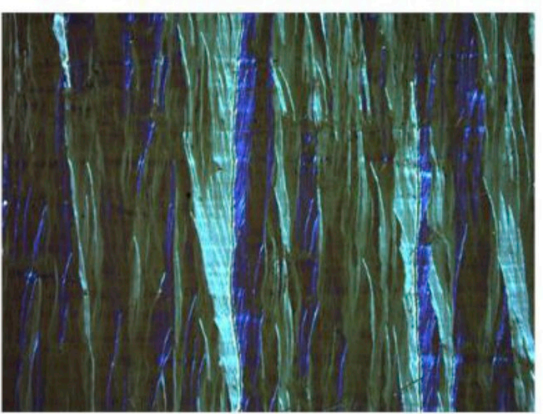

optical image of the sample in bright field (top right) and in cross polarization (bottom right).

sample preparations [51].

In order to probe the degree of order at a microscopic level by the spectroscopic technique, we made use of polarized experiments, acting on both the direction of polarization on the sample and that of the scattered radiation from the film. In the Raman polarized measurements, shown in Fig. 6, the film was thus oriented either parallel or perpendicular to the shearing direction, while the polarizations of the exciting and scattered field were set as shown in the Figure.

The outcome of Fig. 6 is quite remarkable, because all polarized Raman profiles of the film appear as those of the (001) face of the single crystal of the $\alpha$-polymorph of Fig. 4, thus indicating a high degree of iso-orientation of the crystal domains, with the $a$ axis aligned with the shearing direction. To confirm this, the experimental profiles in the Figure are compared to the corresponding DFT calculated polarized spectra. Specifically, the diffraction patterns of the film identify the molecular organization on the substrate, and the polarized Raman spectra also show that this organization is ordered and iso-oriented microscopically, as though the deposited films behaved like a single crystal. Even though the responses of a single crystal perfect lattice and of a film are physically different, what is here observed shows a matching that fully comply with the expectations given by the BAMS deposition of obtaining well oriented films.

Finally, Fig. 6 also demonstrates how the results of the DFT simulated Raman spectra of oriented crystals can be employed to fit the experimental data to account for the spread of the $a b$ plane alignment on the shearing direction, already probed by the rotating GIXD measurements. Details of the procedure are given in the SI; the approach consists in fitting the experiments to a superposition of spectra calculated for single crystals oriented with a Gaussian distribution of angles around the shearing direction. The best fit yields for the distribution a standard deviation $\sigma=21^{\circ}$, which is in excellent agreement with the GIXD result. A slight mismatch between experiments and calculations can be accounted for by some mosaicity of the sample, exposing faces different from the (001).

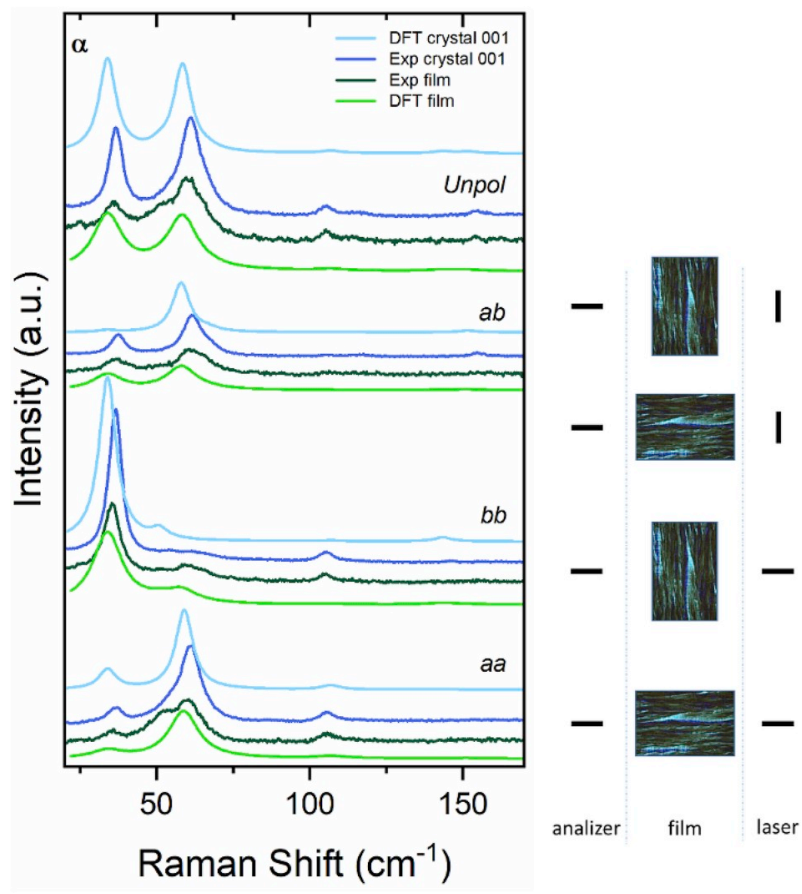

Fig. 6. Polarized Lattice Phonon Raman Spectra of $\alpha$-thioindigo film obtained by BAMS from anisole solution. The sample has been aligned with respect to the shearing direction as shown in the right hand side of the Figure, where the black bars give the direction of polarization for the exciting laser light and for scattered light as selected by the analyser. The film spectra are compared to the corresponding ones measured on the (100) face of a single crystal, to the DFT simulations for the same face and to the spectra resulting from the fit where a Gaussian distribution of crystal orientations about the shearing direction is assumed. 
To summarize, the films obtained with the BAMS procedure showed the presence of well oriented $\alpha$-phase only. Examples of surfaces stabilizing selectively a polymorphic modification are now numerous [47,48,52-56], but less common is combination of selectivity and a high degree of iso-orientation.

\section{Conclusions}

In this work we have dealt with the polymorphism of thioindigo, with the specific purpose of defining the phases present in growths at solid interfaces. This involved the structural and spectroscopic characterization of its bulk phases. For these we have found that, similarly to indigo, it is the polymorph previously thought to be the less stable (i.e. the $\beta$ form), which is instead present in the commercial product and is obtained in most of the common growth methods, such as sublimation or solvent recrystallization. The $\alpha$ form can be easily detected on surfaces, where it grows together with $\beta$ without forming mixed phases. Due to the close energies of the two phases, DFT calculations cannot provide information on their relative stability, but experimental clues in this work and from the literature suggest that the $\alpha$ phase might be metastable at room temperature. The piece of information we gather from the present data is that the $\alpha$ polymorph is however stabilized in growths on a substrate, to the point of becoming the only phase present. The effort of searching for substrate-mediated crystal structures has paved the way to a new approach to address the problem of polymorphism, and the capability of substrates to tune nucleation has been addressed, although the underlying mechanisms are not really understood. The effect of the substrate can be that of a template for molecular deposition [55] or, even more interestingly, the subtle interplay of weak interactions at the interface may eventually lead to fully new molecular arrangements called surface selected polymorphs. Finally, the surface may stabilize a form otherwise metastable in the conditions of growth, and this appears to be the case with thioindigo.

In our study we have applied a deposition method (BAMS), which intrinsically supplies a control of the molecular alignment, on thioindigo dissolved in anisole, obtaining films of the pure $\alpha$ phase, macroscopically oriented along the $a$ axis, which represents the shearing direction of the solution deposited on the hot plate. Polarized Raman and GIXD results confirm the high iso-orientation of the film structures, reminiscent of that of a (001) plane of the single crystal. Given the surface selectivity for this phase, it is likely that the charge transport performances reported for devises fabricated with thioindigo must be ascribed to this polymorph.

\section{Acknowledgements}

We thank CINECA Supercomputing Center for providing computer time through the ISCRA scheme (project C - HP10CA2TWT). The authors thank the Networking Research Center on Bioengineering, Biomaterials and Nanomedicine (CIBER-BBN), the DGI (Spain) project, FANCY CTQ2016-80030-R, the Generalitat de Catalunya (2017-SGR918) and the Spanish Ministry of Economy and Competitiveness, through the 'Severo Ochoa' Programme for Centres of Excellence in R\& D (SEV- 2015-0496). We acknowledge the Elettra Synchrotron Trieste for beamtime allocation and thank Luisa Barba for assistance in using beamline XRD1. T.S. acknowledges the H2020-MSCA-COFUND-2014 Programme (P-SPHERE, Grant agreement 665919).

\section{Appendix A. Supplementary data}

Supplementary data to this article can be found online at https:// doi.org/10.1016/j.dyepig.2019.107847.

\section{References}

[1] Głowacki ED, Voss G, Demirak K, Havlicek M, Sünger N, Okur AC, et al. A facile protection-deprotection route for obtaining indigo pigments as thin films and their applications in organic bulk heterojunctions. Chem Commun 2013;49:6063-5. https://doi.org/10.1039/c3cc42889c.

[2] Uno H, Moriyama K, Ishikawa T, Ono N, Yahiro H. Thioindigo precursor: control of polymorph of thioindigo. Tetrahedron Lett 2004;45:9083-6. https://doi.org/10. 1016/j.tetlet.2004.10.028.

[3] Salzillo T, D'Agostino S, Rivalta A, Giunchi A, Brillante A, Della Valle RG, et al. Structural, spectroscopic, and computational characterization of the concomitant polymorphs of the natural semiconductor indigo. J Phys Chem C 2018;122:18422-31. https://doi.org/10.1021/acs.jpcc.8b03635.

[4] Salzillo T, Rivalta A, Castagnetti N, D'agostino S, Masino M, Grepioni F, et al. Spectroscopic identification of Quinacridone polymorphs for organic electronics. CrystEngComm 2019;21:3702-8. https://doi.org/10.1039/C9CE00070D.

[5] von Helle H. Structure de colorants indigoïdes. IV-Structure cristalline du thioindigo. Bull Soc Chim Fr 1955:1438.

[6] Haase-Wessel W, Ohmasa M, Thioindigo Süsse P. Crystal structural data for modification II. Naturwissenschaften 1977;64:435

[7] Irimia-Vladu M, Gåowacki ED, Troshin PA, Schwabegger G, Leonat L, Susarova DK, et al. Indigo - a natural pigment for high performance ambipolar organic field effect transistors and circuits. Adv Mater 2012;24:375-80. https://doi.org/10.1002/ adma.201102619.

[8] Khusayfan NM, El-Zaidia EFM, El-Nahass MM. Fabrication and electrical characteristics of thioindigo/silicon heterojunction. Silicon 2018;10:2519-26. https:// doi.org/10.1007/s12633-018-9786-3.

[9] Pfattner R, Bromley ST, Rovira C, Mas-Torrent M. Tuning crystal ordering, electronic structure, and morphology in organic semiconductors: tetrathiafulvalenes as a model case. Adv Funct Mater 2016;26:2256-75. https://doi.org/10.1002/adfm. 201502446.

[10] Bedoya-Martínez N, Schrode B, Jones AOF, Salzillo T, Ruzié C, Demitri N, et al. DFT-assisted polymorph identification from lattice Raman fingerprinting. J Phys Chem Lett 2017;8:3690-5. https://doi.org/10.1021/acs.jpclett.7b01634.

[11] Bedoya-Martínez N, Giunchi A, Salzillo T, Venuti E, Della Valle RG, Zojer E. Toward a reliable description of the lattice vibrations in organic molecular crystals: the impact of van der Waals interactions. J Chem Theory Comput 2018;14:4380-90. https://doi.org/10.1021/acs.jctc.8b00484.

[12] Salzillo T, Giunchi A, Masino M, Bedoya-Martínez N, Della Valle RG, Brillante A, et al. An alternative strategy to polymorph recognition at work: the emblematic case of coronene. Cryst Growth Des 2018;18:4869-73. https://doi.org/10.1021/ acs.cgd.8b00934.

[13] Socci J, Salzillo T, Della Valle RG, Venuti E, Brillante A. Fast identification of rubrene polymorphs by lattice phonon Raman microscopy. Solid State Sci 2017;71:146-51. https://doi.org/10.1016/j.solidstatesciences.2017.07.015.

[14] Della Valle RG, Venuti E, Salzillo T, Brillante A, Masino M, Girlando A. Simulated Raman spectra of four tetraphenylbutadiene polymorphs. Int J Quantum Chem 2018;118:1-8. https://doi.org/10.1002/qua.25503.

[15] Del Pozo FG, Fabiano S, Pfattner R, Georgakopoulos S, Galindo S, Liu X, et al. Single crystal-like performance in solution-coated thin-film organic field-effect transistors. Adv Funct Mater 2016;26:2379-86. https://doi.org/10.1002/adfm.201502274.

[16] Georgakopoulos S, Del Pozo FG, Mas-Torrent M. Flexible organic transistors based on a solution-sheared PVDF insulator. J Mater Chem C 2015;3:12199-202. https:// doi.org/10.1039/c5tc02488a.

[17] Temiño I, Del Pozo FG, Ajayakumar MR, Galindo S, Puigdollers J, Mas-Torrent M. A rapid, low-cost, and scalable technique for printing state-of-the-art organic fieldeffect transistors. Adv Mater Technol 2016;1:1-7. https://doi.org/10.1002/admt. 201600090.

[18] Galindo S, Tamayo A, Leonardi F, Mas-Torrent M. Control of polymorphism and morphology in solution sheared organic field-effect transistors. Adv Funct Mater 2017;27:1-9. https://doi.org/10.1002/adfm.201700526.

[19] Riera-Galindo S, Tamayo A, Mas-Torrent M. Role of polymorphism and thin-film morphology in organic semiconductors processed by solution shearing. ACS Omega 2018;3:2329-39. https://doi.org/10.1021/acsomega.8b00043.

[20] Lausi A, Polentarutti M, Onesti S, Plaisier JR, Busetto E, Bais G, et al. Status of the crystallography beamlines at Elettra. Eur Phys J Plus 2015;130. https://doi.org/10. 1140/epjp/i2015-15043-3.

[21] Kabsch W. XDS. Acta Crystallogr Sect D Biol Crystallogr 2010;66:125-32. https:// doi.org/10.1107/S0907444909047337.

[22] Sheldrick GM. SHELXT - integrated space-group and crystal-structure determination. Acta Crystallogr Sect A Found Crystallogr 2015;71:3-8. https://doi.org/10. 1107/S2053273314026370.

[23] Sheldrick GM. Crystal structure refinement with SHELXL. Acta Crystallogr Sect C Struct Chem 2015;71:3-8. https://doi.org/10.1107/s2053229614024218.

[24] Emsley P, Cowtan K. Coot: model-building tools for molecular graphics. Acta Crystallogr Sect D Biol Crystallogr 2004;60:2126-32. https://doi.org/10.1107/ S0907444904019158.

[25] Spek AL. Structure validation in chemical crystallography. Acta Crystallogr Sect D Biol Crystallogr 2009;65:148-55. https://doi.org/10.1107/S090744490804362X.

[26] Sheldrick GM. SHELX-97, program for crystal structure determination 1997.

[27] Macrae CF, Bruno IJ, Chisholm JA, Edgington PR, McCabe P, Pidcock E, et al. Mercury CSD 2.0 - new features for the visualization and investigation of crystal structures. J Appl Crystallogr 2008;41:466-70. https://doi.org/10.1107/ s0021889807067908.

[28] Schrode B, Pachmajer S, Dohr M, Röthel C, Domke J, Fritz T, et al. GIDVis : a comprehensive software tool for geometry-independent grazing-incidence X-ray diffraction data analysis and pole-figure calculations. J Appl Crystallogr 2019;52:683-9. https://doi.org/10.1107/S1600576719004485.

[29] Salzmann I, Resel R. STEREOPOLE : software for the analysis of X-ray diffraction 
pole figures with IDL. J Appl Crystallogr 2004;37:1029-33. https://doi.org/10. 1107/s002188980402165x.

[30] Kresse G, Hafner J Ab. Initio molecular dynamics for liquid metals. Phys Rev B 1993;47:558-61.

[31] Kresse G, Furthmüller J, Hafner J. Ab initio molecular-dynamics simulation of the liquid-metal-amorphous-semiconductor transition in germanium. Phys Rev B 1994;49:14251-69. https://doi.org/10.1016/0927-0256(96)00008-0.

[32] Kresse G, Furthmüller J. Efficiency of ab-initio total energy calculations for metals and semiconductors using a plane-wave basis set. Comput Mater Sci 1996;6:15-50. https://doi.org/10.1016/0927-0256(96)00008-0.

[33] Kresse G, Furthmüller J. Efficient iterative schemes for ab initio total-energy calculations using a plane-wave basis set. Phys Rev B 1996;54:11169-86.

[34] Perdew John P, Burke Kieron, Ernzerhof Matthias. Generalized gradient approximation made simple. Phys Rev Lett 1996;77:3865-8. https://doi.org/10.1103/ PhysRevLett.78.1396.

[35] Blöchl PE. Projector augmented-wave method. Phys Rev B 1994;50:17953-79. https://doi.org/10.1103/PhysRevB.50.17953.

[36] Kresse G, Joubert D. From ultrasoft pseudopotentials to the projector augmentedwave method. Phys Rev B 1999;59:1758-75. https://doi.org/10.1103/PhysRevB. 59.1758.

[37] Grimme S, Ehrlich S, Goerigk L. Effect of the damping function in dispersion corrected density functional theory. J Comput Chem 2011;32:1456-65. https://doi. org/10.1002/jcc.21759.

[38] Bučko T, Hafner J, Ángyán JG. Geometry optimization of periodic systems using internal coordinates. J Chem Phys 2005;122:124508. https://doi.org/10.1063/1. 1864932.

[39] Togo A, Tanaka I. First principles phonon calculations in materials science. Scr Mater 2015;108:1-5. https://doi.org/10.1016/j.scriptamat.2015.07.021.

[40] Fonari A, Stauffer S. Vasp_raman.py 2013https://github.com/raman-sc/VASP/.

[41] Michalska D, Wysokiński R. The prediction of Raman spectra of platinum(II) an ticancer drugs by density functional theory. Chem Phys Lett 2005;403:211-7. https://doi.org/10.1016/j.cplett.2004.12.096.

[42] Polavarapu PL. Ab initio vibrational Raman and Raman optical activity spectra. J Phys Chem 1990;94:8106-12. https://doi.org/10.1021/j100384a024.

[43] Damen TC, Porto SPS, Tell B. Raman effect in Zinc Oxide. Phys Rev 1966;142:570-4.

[44] Chisholm JA, Motherwell S. COMPACK : a program for identifying crystal structure similarity using distances. J Appl Crystallogr 2005;38:228-31. https://doi.org/10.
$1107 /$ s0021889804027074.

[45] Marom N, Distasio RA, Atalla V, Levchenko S, Reilly AM, Chelikowsky JR, et al Many-body dispersion interactions in molecular crystal polymorphism. Angew Chem Int Ed 2013;52:6629-32. https://doi.org/10.1002/anie.201301938.

[46] Salzillo T, Della Valle RG, Venuti E, Brillante A, Siegrist T, Masino M, et al. Two new polymorphs of the organic semiconductor 9,10-diphenylanthracene: Raman and Xray analysis. J Phys Chem C 2016;120:1831-40. https://doi.org/10.1021/acs.jpcc. 5 b11115.

[47] Rivalta A, Salzillo T, Venuti E, Della Valle RG, Sokolovič B, Werzer O, et al. Bulk and surface-stabilized structures of paracetamol revisited by Raman confocal microscopy. ACS Omega 2018;3:9564-71. https://doi.org/10.1021/acsomega. 8 b01246.

[48] Schrode B, Jones AOF, Resel R, Bedoya N, Schennach R, Geerts YH, et al. The substrate-induced phase of a benzothiophene derivative detected by mid-infrared and lattice phonon Raman spectroscopy. ChemPhysChem 2018;19:993-1000. https://doi.org/10.1002/cphc.201701378.

[49] Long DA. Raman spectroscopy. McGraw-Hill; 1977.

[50] Turrel G, Corset J. Raman microscopy: developments and applications. Elsevier Academic Press; 1996. https://doi.org/10.1117/3.1002081.ch62.

[51] Diao Y, Shaw L, Bao Z, Mannsfeld SCB. Morphology control strategies for solution processed organic semiconductor thin films. Energy Environ Sci 2014;7:2145-59. https://doi.org/10.1039/c4ee00688g.

[52] Artusio F, Pisano R. Surface-induced crystallization of pharmaceuticals and biopharmaceuticals: a review. Int J Pharm 2018;547:190-208. https://doi.org/10. 1016/j.ijpharm.2018.05.069.

[53] Jones AOF, Chattopadhyay B, Geerts YH, Resel R. Substrate-induced and thin-film phases: polymorphism of organic materials on surfaces. Adv Funct Mater 2016;26:2233-55. https://doi.org/10.1002/adfm.201503169.

[54] Röthel C, Ehmann HMA, Baumgartner R, Reischl D, Werzer O. Alteration of texture and polymorph of phenytoin within thin films and its impact on dissolution. CrystEngComm 2016;18:588-95. https://doi.org/10.1039/c5ce01889g.

[55] Salzmann I, Moser A, Oehzelt M, Breuer T, Feng X, Juang ZY, et al. Epitaxial growth of $\pi$-stacked perfluoropentacene on graphene-coated quartz. ACS Nano 2012;6:10874-83. https://doi.org/10.1021/nn3042607.

[56] Beyer P, Breuer T, Ndiaye S, Zykov A, Viertel A, Gensler M, et al. Lattice matching as the determining factor for molecular tilt and multilayer growth mode of the nanographene hexa-peri-hexabenzocoronene. ACS Appl Mater Interfaces 2014;6:21484-93. https://doi.org/10.1021/am506465b. 